\title{
The development and longitudinal evaluation of a wellbeing programme: An organisation case study
}

\author{
Anna Sutton · Maggi Evans · Carol Davies · Cathy Lawson
}

\begin{abstract}
Wellbeing programmes are often initiated in organisations based on an assumption that they will promote employee engagement and performance. But the specific elements of a wellbeing programme are rarely evaluated for their efficacy. This case study reports on the development and refinement of a wellbeing programme at a large multi-site European organisation, analysing the utility of wellbeing offerings and the impact of the programme on employee wellbeing over the course of five years. Results from two internal surveys were analysed. The first, conducted at 18-month intervals between 2009 and 2014, evaluated employee engagement. The second, a tailored wellbeing survey conducted in 2014, measured employee wellbeing and perceptions of the individual wellbeing programme offerings. A number of key findings emerged: the wellbeing programme was highly regarded and reported to have a positive impact on employee engagement, some elements of the programme were better received than others, and there were significant differences in the wellbeing levels of different groups. Regression analyses provide evidence for the need to take account of individual employees' current levels of wellbeing when tailoring a wellbeing programme for them, with current wellbeing predicting up to $11 \%$ of the variance in preference for different elements of the programme. Results highlight some of the complexities that organisations should be aware of when understanding employee wellbeing, including the effect of national culture, job grade and current wellbeing levels. This case study provides insight into the development of a wellbeing programme and evidence for its positive contribution to employee engagement.
\end{abstract}

Keywords: wellbeing, employee engagement, evaluation, workplace

\section{Introduction}

The World Health Organisation (2005) has called on organisations to promote employee wellbeing as part of good corporate practice, recognising that employee wellbeing has an impact on the wider society. This focus on work organisations as sources of wellbeing was prefigured by Seligman and Csikszentmihalyi's (2000) call for the development of "positive institutions" which could, amongst other benefits, foster citizens' wellbeing. Building on this approach, Schulte and Vainio (2010) argue that the benefits of employee wellbeing go far beyond the employing organisation. In their model, workforce wellbeing improves the productivity of the individual worker and the organisation, and ultimately contributes to the productivity of the nation, thereby influencing the wellbeing of the population as a whole. They point out that individual wellbeing does not exist in a "vacuum" but is part of the social context. Helliwell's (2011) case study of the Singapore Prison Service demonstrated this in dramatic fashion, illustrating the importance of the social context in promoting wellbeing in an organisation not traditionally associated with a concern for individual wellbeing. The case study reported here 
follows that theme of an organisation as an enabler of wellbeing, both for the employees in the organisation as well as their families. As Schulte and Vainio (2010) argue, the increase in organisational wellbeing may ultimately benefit wider society.

Far from being merely a benevolent ideal, investment in employee wellbeing at work has demonstrable and wide-ranging effects. Wellbeing programmes are reported to reduce absenteeism and turnover, increase employee satisfaction and productivity, and enhance the company profile (PwC, 2008). One of the major impacts of wellbeing programmes is an increase in employee engagement (Robertson, Birch, \& Cooper, 2012). Engagement is defined as a "positive, fulfilling work-related state of mind," which characterises employees who are active, take initiative and seek out new challenges (Schaufeli, Salanova, González-Romá, \& Bakker, 2002), all of which can have substantial impacts on overall organisational performance.

Wellbeing programmes are therefore seen by many organisations as more than simply an effort to "look after employees": they are key to any strategy for improving engagement and performance. A recent review of research on wellbeing initiatives showed that the most commonly-reported reasons for introducing these programmes were economic: as attempts to increase employee productivity or reduce absence (Carmichael, Fenton, Pinilla Roncancio, Sadhra, \& Sing, 2016). But what elements of these often broadly defined wellbeing programmes are most effective? And how can an organisation ensure that it is offering a wellbeing programme that suits individual employees? In this case study, we report on the development and refinement of a wellbeing programme at a large multi-site European organisation. We consider both the perceived usefulness and value to the employees of individual wellbeing offerings and the impact of the programme on employee engagement over the course of several years. Before describing the case in detail, we review the current literature on wellbeing at work, how "wellbeing" is best defined, how it can be promoted at work, the role of national and cultural differences in wellbeing, and, finally, the potential return on investment.

\section{Wellbeing and work}

"Wellbeing" is a broad concept, and, as such, there is often debate over its definition. Perhaps one of the most definitive explanations is provided by Diener (2000) in a paper calling for national measures of happiness. He regards wellbeing as a subjective evaluation of life: the emotions we feel, the activities we engage in, the balance of pleasurable and painful experiences we have, and our general satisfaction with life. In workplace-based research, some authors opt for a narrow definition which equates wellbeing with job satisfaction (Sousa-Poza \& Sousa-Poza, 2000), while others argue for a more holistic concept of the quality of working life (Schulte \& Vainio, 2010). In a seminal review of the relationship between work, health and wellbeing, Waddell and Burton (2006) distinguished between health and wellbeing, noting that the former is still operationalised in terms of the absence of symptoms or illnesses, while the latter is a subjective state of satisfaction or happiness with one's life. In line with Diener's general definition of wellbeing and the consensus in the occupational psychology literature, we use this broader definition here: seeing wellbeing as a broad concept encompassing our physical, psychological and social health as well as our levels of contentment with our health.

In their review of the work and wellbeing literature, Waddell and Burton (2006) found that good work can promote wellbeing in several ways. For example, it provides workers and their families with the economic resources they need to ensure physical wellbeing and participation in society. Work is also an important source of individual identity and social roles, and employment status has clear links with physical and mental health. In addition, being in work has been found to improve the quality of life of sick and disabled people. In contrast, 
unemployment has strong causal links with poorer mental and physical health. These promising findings need to be understood in context, however, because the relationship between work and wellbeing is complex and two-way (Vickerstaff, Phillipson, \& Wilkie, 2012). While work can be beneficial for wellbeing, it can also have a negative impact. Examples of this latter effect are when the work itself is physically hazardous or when workers suffer stress or trauma at work. Promoting wellbeing at work then, should incorporate both risk reduction as well as wider health promotion.

\section{Individual and national differences in wellbeing}

In understanding the role of wellbeing at work, it is important to take account of individual and national differences. Diener et al (2003) conducted a review of the relationships between personality, culture and subjective wellbeing and found strong evidence that wellbeing varies both between individuals and between countries. For example, individuals with higher extraversion and lower neuroticism had higher subjective wellbeing, though the direction of causality here is yet to be determined. Besides an individual's personality, age has also consistently been shown to have an effect on work wellbeing. This relationship is commonly described as having a U-shape: young workers report high wellbeing and this then tends to drop in the late 20s/early 30s, before climbing again (Warr, 1992). This influence is not explained simply by seniority in the organisation, however: job level has complex relationships with wellbeing, seemingly increasing both job-related anxiety and enthusiasm. A large-scale analysis by Wood (2008) found evidence that greater job control, such as is often found in higher-level jobs, is associated with higher wellbeing, while greater job demands are associated with lower wellbeing, and that increased job control can act as a buffer to the negative impact of demands.

Similarly to individual differences, both nations and ethnic groups within nations were found to differ in wellbeing (Diener et al., 2003). Key influences on these cultural differences included wealth (particularly important for wellbeing for poorer groups), self-enhancement differences between cultures, or a differential value ascribed to happiness itself (e.g., Joshanloo \& Weijers, 2013). National differences in work wellbeing have also been identified, as, for example, in a study comparing 21 mainly European countries (Sousa-Poza \& Sousa-Poza, 2000). This study identified the highest job satisfaction in Denmark, with Eastern European countries and Japan reporting lower levels. Interestingly, in all countries, the level of work wellbeing is reported to be quite high.

These individual and cultural differences in wellbeing indicate two important issues for organisations to bear in mind when developing wellbeing programmes. First, some influences on wellbeing may well be out of the control of the organisation. For example, personality traits and demographic differences between people have been shown to have close relationships with subjective wellbeing (González Gutiérrez, Jiménez, Hernández, \& Puente, 2005). Second, wellbeing programmes that can be tailored to the individual or cultural needs are likely to be more successful than a "one size fits all" approach. Schueller (2012) demonstrated that different types of happiness-improving interventions were more effective for introverts than extroverts, indicating that person-activity fit is important in positive psychology interventions. Being able to tailor wellbeing offerings dependent on the individual's current level of wellbeing or nationality is likely to enhance return on investment: improving the wellbeing of those with both low and high wellbeing. We are able to address this issue of individual and national differences in this case study, as it includes several different European sites. 


\section{Wellbeing provision at work and return on investment}

In a large-scale survey of UK employers, Young and Bhaumik (2011) investigated the importance that employers ascribed to worker wellbeing and the provisions that they made for their staff. They found that nine out of 10 employers believed they had a responsibility to promote their employees' wellbeing and that there was a link between wellbeing and work, and only 18\% believed that wellbeing initiatives did not provide a good return on investment. The top areas of "wellbeing" provision came under the headings of general benefits, provided by $70 \%$ or more of the organisations and including holidays and pensions, or traditional health and safety initiatives, provided by about half of the organisations and including training in injury prevention and workplace adjustments. In addition, the larger the organisation, the greater the number of initiatives to promote wellbeing they reported providing to staff. Small organisations of up to 50 employees reported on average only three out of 20 initiatives, while large organisations (> 250 employees) reported an average of seven initiatives in the private sector and nine in the public sector.

The return on investment for wellbeing programmes tends to be positive: a recent metaanalysis that considered study quality as a factor in estimating ROI suggested an overall return of 1.38 on every US\$ spent (Baxter, Sanderson, Venn, Blizzard, \& Palmer, 2014). However, this ROI does vary dramatically among different organisations. In one report, for example, the benefit-cost ratio across different organisations ranged from 1 to 34 ( $\mathrm{PwC}, 2008)$. While the factors contributing to good ROI are complex, one issue that frequently arises is the need to plan the provision of different elements based on what the employees actually need or value (Spence, 2015). Too often, organisations adopt a haphazard approach to developing wellbeing programmes: investing in a wide range of initiatives without being sure which elements will be effective. In addition, there is rarely any consideration of which elements of a programme might be particularly beneficial for employees with different levels of wellbeing. Instead, it is often hoped that a wellbeing programme will simply improve wellbeing across the board. Having evidence of which elements might be particularly effective for employees with low or high wellbeing, however, would provide organisations with a better basis for their decisions.

In reporting this case study, therefore, we have three main aims. First, we assess the impact of the wellbeing programme on employee engagement and the utility of individual elements. Second, we evaluate the effect of nationality and job seniority on individual wellbeing. And third, we make recommendations for tailoring wellbeing initiatives towards employees with differing levels of wellbeing.

\section{The case}

The BGL Group (https://www.bglgroup.co.uk) is a major financial services company providing several different services, including insurance brands, comparison websites and legal services. It employs over 3,000 people on 11 sites (nine in the UK, one in the Netherlands and one in France). The organisation has top-level commitment to supporting the wellbeing of its employees: the BGL Group strategy explicitly states that corporate social responsibility (CSR) and employee engagement targets are as important as profit and customer satisfaction. The wellbeing programme at BGL is promoted internally and externally as a fundamental part of the organisation's overall strategy, being seen as an integral part of what makes BGL a unique and preferred employer. From the first inception of the wellbeing programme in 2009, it was agreed that supporting employee engagement was "the right thing to do" and it would inherently bring benefits to the organisation. Beyond this, the organisation also promotes itself as a responsible 
company with the motto "Doing right by doing good" and working to improve not just its local community but also contributing to national and international projects to improve wellbeing. In this, the BGL Group can be seen as promoting itself as a "positive institution" (Seligman \& Csikszentmihalyi, 2000): an enabler of employee and wider societal wellbeing.

Alongside this general commitment to investing in employee wellbeing, BGL considers it important to capture some information on the overall impact of the programme and its elements. Following discussion amongst the executive team, it was agreed that increased employee engagement should be the primary measure, rather than any more specific return on investment calculation. Accordingly, employee engagement was tracked through an annual survey, and further "take-up" and "satisfaction" feedback was sought on each element of the programme. This focus on engagement is consistent with the literature, where increased levels of engagement are frequently cited as an outcome of increased employee wellbeing (Robertson, Birch, \& Cooper, 2012).

The programme represented a general wellbeing programme encompassing a wide range of activities (for example, free fruit, access to a gym, access to counselling services, health checks and support with healthier lifestyle choices). While many of these may be considered "wellness" rather than "wellbeing" elements, focusing as they do on the promotion of physical health, the programme overall was concerned with the holistic physical, mental and emotional wellbeing of the employees. We therefore use the term "wellbeing" rather than "wellness" in this paper.

In the first couple of years after its launch, several different elements were added to the programme and subsequently BGL began to review and refine the programme, initiating a more planned approach to meet the needs of a rapidly expanding and diverse workforce. The programme has been developed over the course of five years and continues to be refined and adjusted, based on feedback from the employees. At the time of the survey reported here, a total of 15 individual elements were offered in the overall wellbeing programme, with a further 28 being considered for future inclusion (see Appendix A). These elements ranged from the provision of free fruit to employees, through to subsidised appointments with an osteopath, to access to help with budgeting and financial organisation. Also included in the broad wellbeing programme were legally-required elements such as provision of eyecare vouchers. While some elements were offered across all sites (e.g., free fruit) other elements varied significantly by site. For example, at one site there was an on-site gym, while other sites offered discounted membership to local gyms instead.

Having run the wellbeing programme for nearly five years, the organisation wanted to conduct a full review. There were three key themes that needed to be addressed, the first being an evaluation of the wellbeing programme's effects and perceived value. What was the overall impact of the programme and which elements of the programme seemed to have the greatest impact? Second was an assessment of the employees' wellbeing needs. What were the health and wellbeing issues experienced by the employees and were there any significant differences between employee groups? Third was a proposal for the future changes to the wellbeing programme. What did employees most want from the organisation in terms of support for wellbeing? In tandem with this, the organisation was keen to signal a subtle shift in the wellbeing provision. There was a concern that the offer had been somewhat "paternalistic" in approach, and, moving forward, they wanted a clearer focus on partnership, with a greater emphasis on employee involvement and personal responsibility.

In the following sections, we report on the methodology used to gather and analyse that feedback, and the resultant recommendations that can be made for organisations considering implementing or changing their own wellbeing programmes. 


\section{Method}

The case uses two sets of data: a general engagement survey and a tailored wellbeing survey.

\subsection{Engagement Survey}

Employee engagement was assessed as part of a 60-question internal survey covering a range of issues, such as employees' views of their day-to-day work, their relationships with managers and co-workers and so on. The survey has been administered by PeopleMetrics at 18-month intervals over a five-year period (2009-2014). It was accessed via the company's intranet and launched with an email from the CEO, promoting the importance of the survey, encouraging employees to complete it and assuring them that responses would be anonymous. Response rates are very good: the most recent survey (2014) had a response rate of 93\% (3,053 employees invited and 2,840 responded).

An overall engagement score is calculated from four behaviourally-based outcome items, measured on a five-point Likert-type scale, from "strongly disagree" to "strongly agree":

Retention: It would take a lot to get me to leave BGL.

Effort: Being part of BGL motivates me to go beyond what is expected.

Advocacy: I recommend BGL as a great place to work.

Passion: I really like working for BGL.

For each item, the percentage of employees who agree or strongly agree is noted. The overall engagement score is then calculated as the mean of these percentages.

From 2010, employees were also asked a specific question about wellbeing. Again scored on a five-point agreement scale, the item was worded as "BGL takes the wellbeing of employees seriously."

\subsection{Tailored wellbeing survey}

In a separate survey, 1330 employees from 11 sites rated their personal wellbeing, the current wellbeing initiatives and their preferences for future initiatives. The survey was distributed online via internal email with internal communication to encourage people to take part. The survey was designed so that only services available at the respondent's site were visible to them. This was especially important for the European sites, which had been recently acquired and did not yet have access to the full wellbeing programme.

- Employees rated their wellbeing on a scale of one to five in the following different areas: health, fitness, emotional wellbeing, perceived energy levels and quality of sleep. They were also asked to indicate whether this had changed over the last 12 months.

$\circ$ An overall wellbeing measure was created from the mean of these five items. ( $\alpha$ $=0.82$ )

- The current offerings in the wellbeing programme were evaluated by respondents on three criteria:

- Whether the respondent was aware of the offering

- If aware, how much the respondent had made use of this element

- And if used, to rate the value of the service on a scale of 1 to 5 ( $1=$ not at all useful; 2 = not useful; $3=$ OK; 4 = useful; 5 = extremely useful)

- Finally, respondents were asked to indicate their preference for which of the elements should be included as the wellbeing programme developed in the future.

In addition, demographic information was collected, including work site, country and job grade. The number of employees per individual site ranged from a minimum of four to a maximum of 
449. For analyses involving the size of the site, sites were grouped into small (up to 50 employees), medium (51-200 employees) and large (>200 employees).

The majority of employees (96\%) were based at the nine UK sites, with $2 \%$ in France and $2 \%$ in the Netherlands. Job level was assessed according to four different levels: staff (62\%), team leader (7\%), manager/senior manager (27\%) and associate director or above $(4 \%)$.

\section{Results}

Results from these two surveys were analysed to provide a picture of the wellbeing programme and its effects over time, as well as to make recommendations for further refinements. We report the results using the structure of the three main themes described above.

\subsection{Impact and perceived value of the wellbeing programme}

Figure 1 below demonstrates that over the five-year period of the engagement survey, there was an increase in both employee engagement and the belief that the organisation takes the employees' wellbeing seriously.

Figure 1: Employee engagement

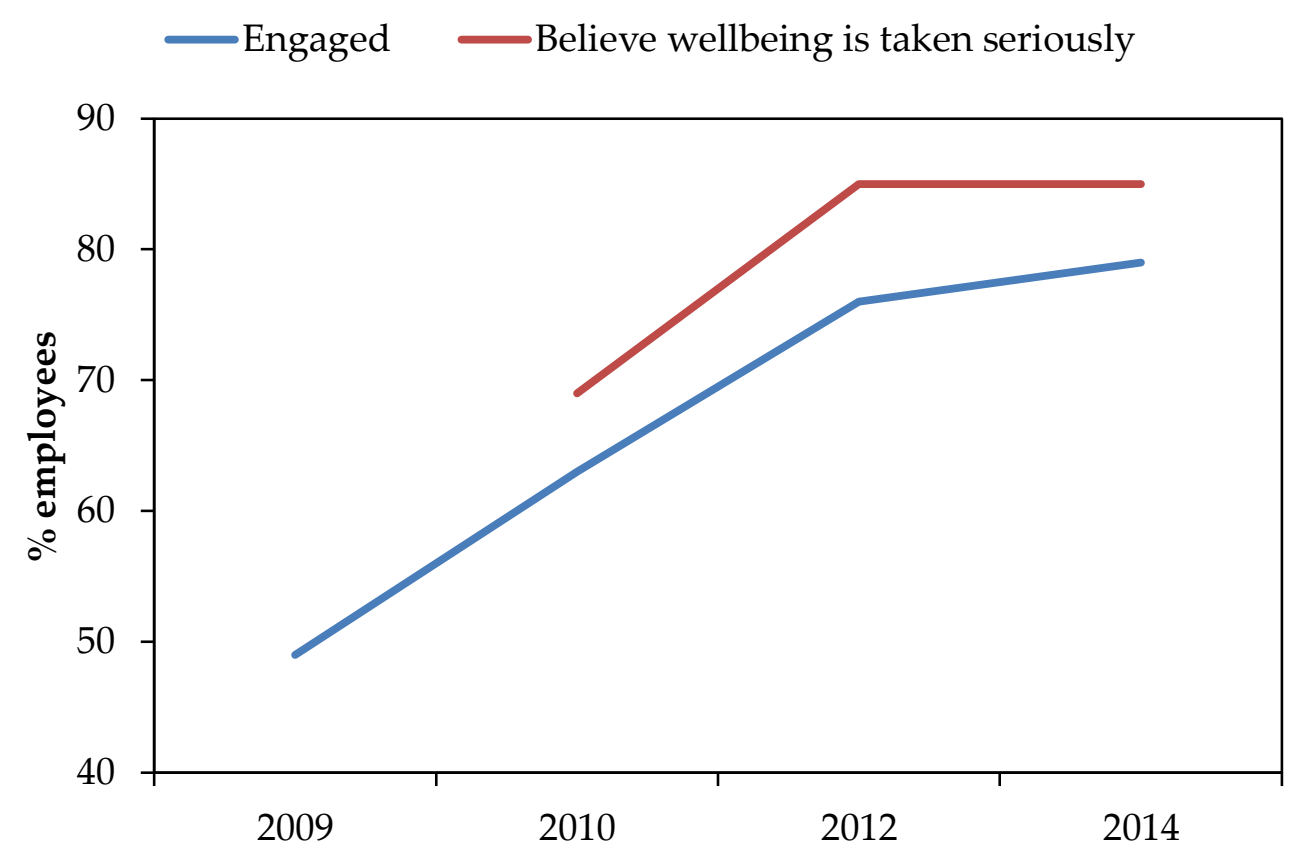

In the Tailored Wellbeing Survey, the five most well-known elements were: free fruit (98.9\% aware), eyecare vouchers (88.1\%), discounted gym membership (85\%), osteopath appointments $(84.6 \%)$ and "know your numbers," an in-house health check programme $(79 \%)$.

Respondents who indicated they knew about the element were then asked to indicate how often they used it. The highest usage rates (using the service "whenever possible") were for free fruit $(66 \%)$ and "know your numbers" (36\%). Eyecare vouchers, healthy eating options in the restaurant, discounted gym membership, at desk massage and private healthcare membership were used "whenever possible" by between 22 and 28\% of respondents. Meanwhile, the least used services were a second opinion medical referral scheme and a dental/hospital cash plan (both 6\%), weight watchers meetings (9\%) and organised lunchtime walks (12\%). 
Finally, those respondents who had used a service were asked to rate its usefulness or value to them. The mean value ratings for each element of the wellbeing programme are illustrated in Table 1 below ( $1=$ not at all useful to $5=$ extremely useful $)$.

Table 1: Mean value ratings for wellbeing programme elements

\begin{tabular}{lc}
\hline Service & Mean value rating \\
\hline Discounted gym membership & 4.7 \\
Free fruit & 4.6 \\
At desk massage & 4.6 \\
Eyecare vouchers & 4.6 \\
Private healthcare & 4.6 \\
Health screening & 4.5 \\
Remedial sports massage & 4.2 \\
Know your numbers (in-house health checks) & 4.1 \\
Healthy eating options in restaurant & 4.1 \\
Osteopath & 4.0 \\
Employee Assistance Programme (telephone counselling) & 3.9 \\
Organised lunchtime walking & 3.8 \\
Dental/hospital cash plan & 3.8 \\
Best doctors (second opinion medical referral) & 3.5 \\
Weightwatchers site meetings & 3.2 \\
\hline
\end{tabular}

Overall, the findings indicated that BGL was running a successful wellbeing programme, with high awareness and usage across much of the offer, and those who used the services tended to be very positive about them. Some services had high awareness and were highly rated, but were used less, for example, the discounted gym membership, the EAP, the osteopath and remedial sports massage.

\subsection{Employee wellbeing}

There is no benchmark data available to see how the BGL ratings compare with other organisations, however, moving forward there is an opportunity to use the figures to monitor changes within the BGL population and to link it to sickness absence data. Key general findings were:

- Overall health was the highest rated of the wellbeing elements and a fifth of respondents reported that their health had improved in the preceding 12 months

- There were lower scores for fitness, energy and sleep, and, across the five wellbeing elements, a small but significant minority $(17 \%)$ of the respondents reported low ratings (1 or 2 out of 5 ).

- Sleep emerged as a concern across the organisation, with $27 \%$ of people scoring their sleep as a 1 or 2 and $25 \%$ stating it had got worse over the past 12 months.

To investigate the effect of national site on wellbeing, a one-way ANOVA was conducted on the overall wellbeing scores. There was a significant main effect of country $(F(2,1326)=4.07, \mathrm{p}<$ $\left..05, \omega^{2}<0.01\right)$ on wellbeing. Bonferroni post-hoc tests indicated that Dutch employees $(\mathrm{M}=3.78$, $\mathrm{SD}=.76)$ had significantly higher wellbeing than British employees $(\mathrm{M}=3.36, \mathrm{SD}=.74)$, with a moderate effect size $(d=.56)$. This difference is in line with previous research showing work wellbeing as higher in the Netherlands than the UK (Sousa-Poza \& Sousa-Poza, 2000). There 
were no significant differences between France $(M=3.44, S D=.91)$ and either of the other countries.

Similarly, a one-way ANOVA revealed a significant main effect of organisational size on employee wellbeing $\left(F(2,1327)=9.62, p<.001, \omega^{2}=0.013\right)$. Post-hoc tests demonstrated that employees on small sites had higher wellbeing $(\mathrm{M}=3.70, \mathrm{SD}=.81)$ than those at both medium $(\mathrm{M}=3.30, \mathrm{SD}=.73, d=.53)$ and large sites $(\mathrm{M}=3.37, \mathrm{SD}=.74, d=.44)$, both with moderate effect sizes.

Wellbeing showed a general trend of increased wellbeing with seniority (Figure 2 below). A one-way ANOVA revealed a significant main effect for seniority $\left(F(3,1326)=9.7, p<.001, \omega^{2}=\right.$ $0.02)$, with Bonferroni post-hoc tests indicating that directors $(\mathrm{M}=3.65, \mathrm{SD}=.62)$ had significantly higher wellbeing than staff $(\mathrm{M}=3.30, \mathrm{SD}=.79, d=.45)$ and team leaders $(\mathrm{M}=3.28, \mathrm{SD}=.77, d=$ $.51)$, while managers $(\mathrm{M}=3.51, \mathrm{SD}=.64)$ had significantly higher wellbeing than staff $(d=.28)$.

Figure 2: Wellbeing and job seniority

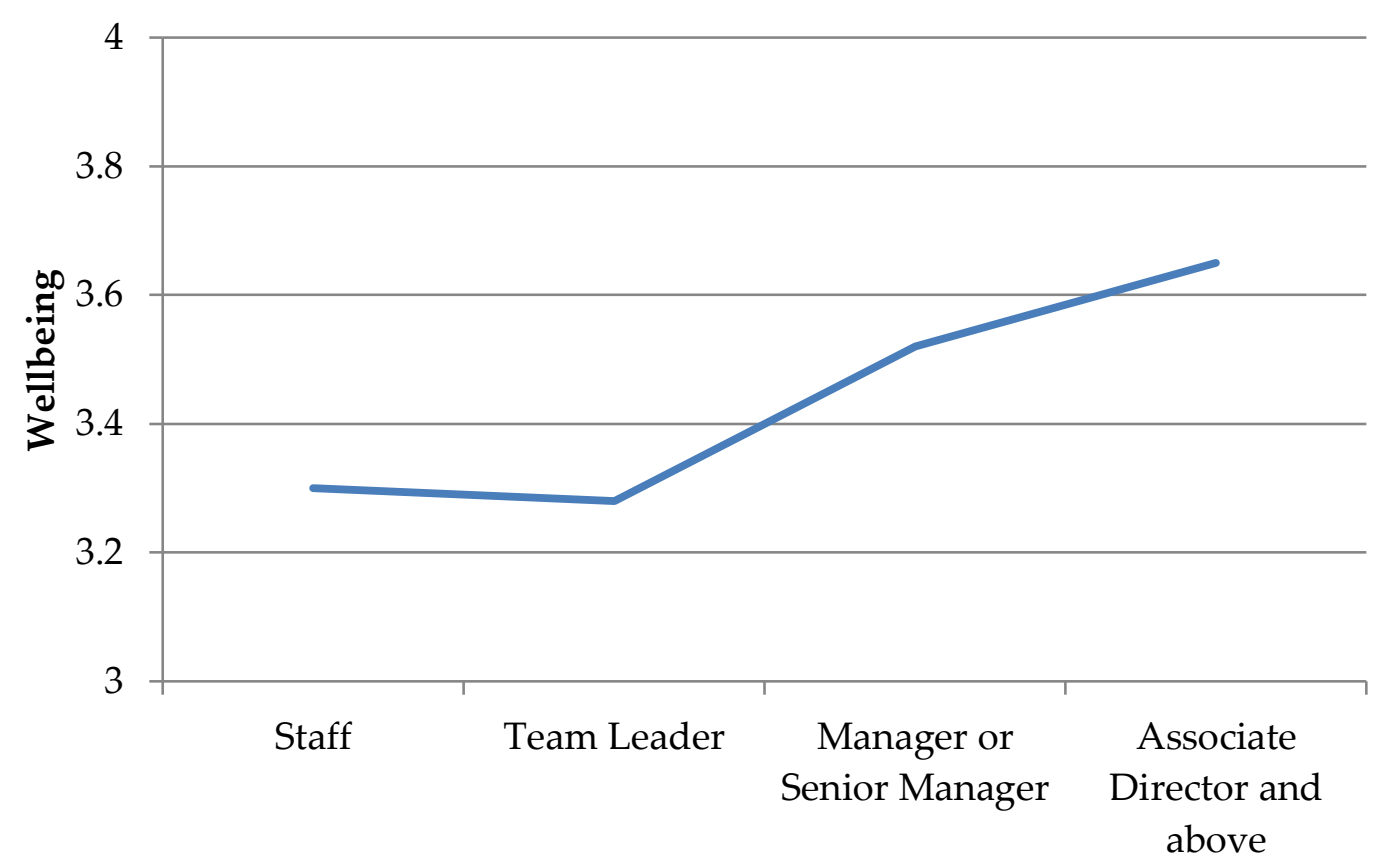

\subsection{Future developments}

Regression analyses were conducted to identify whether employees' current levels of wellbeing influenced their preference for the inclusion of specific elements in the wellbeing programme in the future. Here, we used the specific measures of wellbeing (physical health, fitness, emotional wellbeing, sleep and energy) as predictors, and the individual wellbeing elements as outcomes. Level of wellbeing significantly predicted employees' choice of programme element for 13 of the items. Details of the model fit and significant predictors are shown in Table 2 below.

These analyses provide evidence for the need to take account of employees' current levels of wellbeing when tailoring a wellbeing programme towards them, as wellbeing predicted between 1 and $11 \%$ of the variance in preference for different elements. Figure 3 below helps to illustrate this by providing a visual summary of the types of programme elements that employees would like to see more of, predicted by their current wellbeing levels. 
Table 2: Regression analyses

\begin{tabular}{lcclc}
\hline Programme element & $\mathbf{R}^{2}$ & $\mathbf{F}(\mathbf{5}, \mathbf{1 3 2 4})=$ & Predictors & $\beta$ \\
\hline Organised team sports & .03 & $9.03^{* * *}$ & Fitness & $.18^{* * *}$ \\
Subsidised gym membership & .01 & $3.67^{* *}$ & Fitness & $.08^{*}$ \\
Weight management & & & Emotional & $.09^{*}$ \\
Anger management & .05 & $12.43^{* * *}$ & Health & $-.08^{*}$ \\
Changing facilities & & & Fitness & $-.16^{* * *}$ \\
Stress management support & .02 & $4.16^{* *}$ & Fitness & $.12^{* *}$ \\
& .03 & $8.87^{* * *}$ & Fitness & $.19^{* * *}$ \\
Quitting smoking help & .08 & $23.97^{* * *}$ & Energy & $.09^{*}$ \\
Sleep clinic & & & Emotional & $-.31^{* * *}$ \\
& .01 & $3.08^{* *}$ & Health & $-.10^{* *}$ \\
Managing anxiety & .11 & $32.25^{* * *}$ & Health & $.07^{*}$ \\
At desk massage & & & Sleep & $-.38^{* * *}$ \\
Healthy lifestyle information & .08 & $23.31^{* * *}$ & Emotional & $-.30^{* * *}$ \\
Help with energy levels & .01 & $2.70^{*}$ & Fitness & $-.11^{* *}$ \\
Help with fatigue & .01 & $3.28^{* *}$ & Fitness & $-.07^{*}$ \\
& .04 & $11.53^{* * *}$ & Energy & $-.21^{* * *}$ \\
& & & Sleep & $-.12^{* *}$ \\
\hline p & .04 & $10.41^{* * *}$ & Energy & $-.15^{* * *}$ \\
& & & Sleep & $-.13^{* * *}$ \\
& & & Emotional & $.08^{*}$ \\
\hline
\end{tabular}

${ }^{*} \mathrm{p}<0.05,{ }^{* *} \mathrm{p}<0.01,{ }^{* * *} \mathrm{p}<0.001$

Figure 3: Current wellbeing as a predictor of preferences for future programme elements

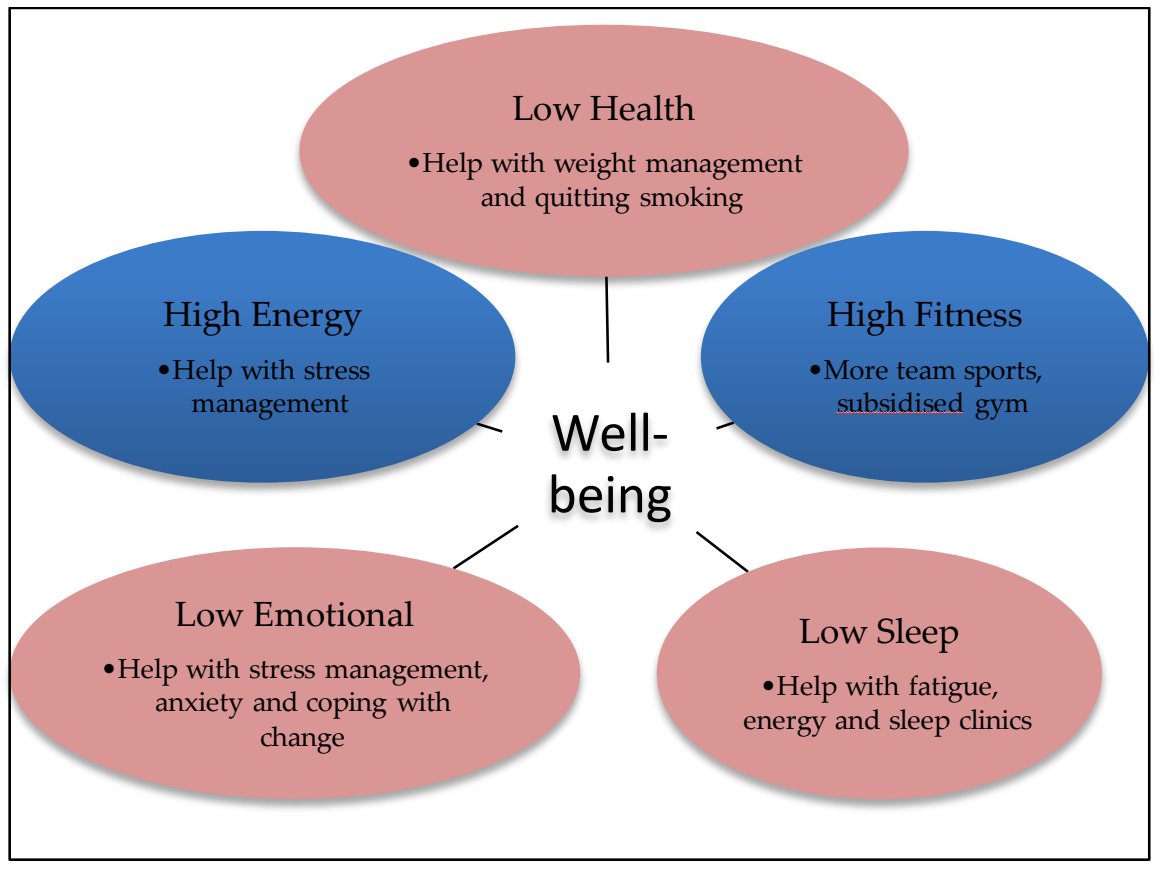




\section{Discussion}

The study presents a rare insight into the planning, implementation and development of a complete wellbeing programme in a private, multi-site European organisation, with longitudinal measures demonstrating its impact over a five-year period. Results over that period provide evidence for the positive impact of a wellbeing programme on employee engagement. Additionally, the introduction of this wellbeing programme has encouraged employees' belief that their organisation takes their wellbeing seriously. This in itself is a positive outcome for the organisation, indicating that the employees feel valued by their employer, before any impacts on wellbeing itself.

Analysis of the employee wellbeing ratings highlighted some important issues for the BGL Group to be aware of as their programme develops. Although overall wellbeing ratings were generally high, there was a significant minority of employees who reported lower ratings, and these can be expected to impact on health and absence in the longer term. Of particular interest here is the concern expressed over sleep by a quarter of employees. Given the high level of reported sleep difficulty, it was surprising that the sleep clinic element of the wellbeing programme did not gain greater support. It could be that respondents were not clear what was meant and feared having to sleep in a research laboratory rather than attending a workshop to discuss lifestyle or habit changes that could improve sleep. In this case, it may be that simply providing clearer information on this option, and encouragement for take-up could lead to an improvement in wellbeing for a substantial group of employees. For organisations planning to implement or improve a wellbeing programme, this finding highlights the importance of effective communication for the full benefits of wellbeing offerings to be experienced by employees.

The results also illustrate some of the complexities that organisations need to be aware of when measuring and interpreting their employees' wellbeing. First, it is important to take account of national differences in reported wellbeing when planning developments for a wellbeing programme. In this study, employees in the Netherlands reported higher wellbeing than those in the UK, which is in line with previous research (Sousa-Poza \& Sousa-Poza, 2000) that indicated that these national differences are at least partly explicable by differences in workrole inputs and outputs. In interpreting national differences in wellbeing, then, organisational decision-makers should be aware that UK employees are likely to report slightly lower levels of wellbeing than many other countries, and that this could well be independent of any wellbeing programme.

Second, it is worth noting how wellbeing in this organisation was related to the employee's job grade, with wellbeing increasing with seniority. This contrasts with previous work which identified a complex relationship of job seniority and wellbeing, with seniority increasing both job-related enthusiasm and anxiety (Warr, 1992). Wood's work (2008) indicates that this paradoxical effect may be due to an increase in both job control and job demands in senior positions. There are two possible explanations for the finding in this case study that seniority is associated solely with better wellbeing. The first is that the measure of wellbeing used here does not tap the anxiety-contentment axis of job related wellbeing (Warr, 1990). An alternative explanation is that the BGL Group may be supporting higher level managers well, counteracting the increased anxiety that is common at this level. While the exact mechanism underlying this achievement is not yet known, a possible explanation may be that it is the specific wellbeing offerings that are available solely to higher level managers (for example, access to private healthcare) that enable this increased wellbeing. For organisations wishing to provide tailored 
wellbeing offerings to their employees, it is worth considering the extent to which their offerings may provide a buffer to the increased demands of senior jobs.

Finally, the results reported here can help organisations to develop a wellbeing programme which incorporates elements designed to appeal to employees with different levels of wellbeing. Providing employees with a wellbeing programme which actually meets their needs is an essential step in ensuring that participation in the programme is increased (Spence, 2015). Rather than providing a one-size-fits-all wellbeing programme, this case study provides evidence that it is possible to provide elements tailored to specific needs. There are two potential applications of these findings. First, for organisations with limited funds, or those that wish to ensure that their wellbeing programme is targeting specific groups of employees, elements could be chosen which appeal specifically to that group. For example, a workforce that was found to be particularly low on emotional wellbeing would benefit from help in dealing with change, while a workforce reporting high levels of energy would appreciate help with stress management. Alternatively, a wellbeing programme could be developed to include elements which address each of the five areas of wellbeing assessed in this case, allowing employees to engage in whichever offerings they felt would be beneficial. This latter approach ensures that there are offerings which are likely to appeal to a range of different employees, rather than unwittingly providing a wellbeing programme which would only appeal to employees with, say, low levels of emotional wellbeing.

\subsection{Limitations}

As with all case studies, the findings reported here are specific to a single organisation, and care should be taken when generalising to other organisations and industries. However, the detail which has been provided about the organisation itself will hopefully enable practitioners to draw conclusions about the applicability of these findings to their own organisations, while also providing clear illustrations of some of the challenges and opportunities in implementing wellbeing programmes.

While we had information about levels of engagement in the organisation over the course of the programme implementation, the evaluation of the wellbeing programme currently lacks links to "harder" HR measures such as absence or turnover. Other studies have demonstrated these links very well, however $(\mathrm{PwC}, 2008)$, and there is no reason to suppose they do not hold true for this case study too.

Finally, in considering the implementation of wellbeing programmes in other organisations, a note of caution should be raised. Employee wellbeing is of central priority to the BGL Group and is emphasised both in the organisation's formal strategy and in its culture. An organisation which does not prioritise wellbeing to this extent may well find that results of a wellbeing programme would differ substantially.

Wellbeing programmes often build on an organisation's traditional benefits or health and safety initiatives (Young \& Bhaumik, 2011) rather than being developed from a theoretically sound understanding of the elements of wellbeing. This case study reports on the efficacy of one such "ad-hoc" approach but it would be interesting to compare this approach with a wellbeing programme that was developed from a comprehensive model, such as the Five Ways to Wellbeing (Aked, Marks, Cordon, \& Thompson, 2008).

\subsection{Impact}

The BGL Group has used the results of these surveys to guide action at two levels: local and group. Local teams are encouraged to discuss the results in focus groups and to understand 
them more fully, specifically to decide what actions they can take in relation to various questions. Each area also has a champion who helps to co-ordinate actions and help them happen. The champions meet as a collective across the Group once a quarter to maintain momentum and share best practice.

At Group level, the focus is on the key issues from the survey that appear to be affecting most employees. With such high engagement scores, it can be a challenge not to get drawn into tiny things but still to respond in a way that improves employees' engagement and wellbeing. With this in mind, a 52-week programme was created called "Pulse Little Things," that addressed some of these frustrations. These included better lighting in the car parks and more racks for bikes. There were also a few larger issues that appeared to be consistently being flagged, including dress code, flexible working and career development. These three topics were discussed by the board and solutions identified for each, which were then rolled out during the remainder of the year.

In addition, the 18-month interval between surveys was considered to be too long a time to demonstrate the benefits of any initiatives and also keep to pace with changing opinions. The Group therefore introduced quarterly "temperature checks" across a sample group. This provides indications of whether certain areas are improving, reducing or staying the same, so that the champions can keep abreast of developments throughout the year.

BGL is pleased with the engagement level they have but wish to continue to improve, and have set a stretching target of $85 \%$ engagement by 2017 . They are continuing to implement the various initiatives that have been promised (e.g., at desk massage for the legal services business, improved gym discounts for all sites, reviewing the catering offering to ensure that it aligns with a healthy balanced diet). The Group is now considering in depth what they want on their engagement agenda, and is developing plans for how this can be achieved.

\section{Conclusion}

This case study has demonstrated three main points. First, it has evaluated the utility of individual elements of a wellbeing programme in a large multi-site organisation, identifying which of them are most appreciated by employees. Second, it has illustrated the effect of a wellbeing programme on enhancing employee engagement. And finally, it has made recommendations for tailoring wellbeing programme elements to employees with differing levels of wellbeing.

\section{Authors}

Anna Sutton

Manchester Metropolitan University

a.sutton@mmu.ac.uk

Maggi Evans

Mosaic Consulting

Carol Davies

National House Building Council

Cathy Lawson

BGL Group 


\section{Publishing Timeline}

Received 25 November 2015

Accepted 11 April 2016

Published 14 May 2016

\section{References}

Aked, J., Marks, N., Cordon, C., \& Thompson, S. (2008). Five ways to wellbeing: The evidence. http://www.neweconomics.org/publications/entry/five-ways-to-well-being-the-evidence

Baxter, S., Sanderson, K., Venn, A. J., Blizzard, C. L., \& Palmer, A. J. (2014). The relationship between return on investment and quality of study methodology in workplace health promotion programs. American Journal of Health Promotion, 28(6), 347-363. http://dx.doi.org/10.4278/ajhp.130731-LIT-395

Carmichael, F., Fenton, S.-J., Pinilla Roncancio, M., Sadhra, S., \& Sing, M. (2016). Workplace wellbeing programmes and their impact on employees and their employing organisations: A scoping review of the evidence base. http://epapers.bham.ac.uk/2103/

Diener, E. (2000). Subjective well-being: The science of happiness and a proposal for a national index. American Psychologist, 55(1), 34-43. http://dx.doi.org/10.1037/0003-066X.55.1.34

Diener, E., Oishi, S., \& Lucas, R. E. (2003). Personality, culture, and subjective well-being: Emotional and cognitive evaluations of life. Annual Review of Psychology, 54(1), 403-425. http://dx.doi.org/10.1146/annurev.psych.54.101601.145056

González Gutiérrez, J. L., Jiménez, B. M., Hernández, E. G., \& Puente, C. P. (2005). Personality and subjective well-being: Big five correlates and demographic variables. Personality and Individual Differences, 38(7), 1561-1569. http://dx.doi.org/10.1016/j.paid.2004.09.015

Helliwell, J. F. (2011). Institutions as enablers of wellbeing: The Singapore prison case study. International Journal of Wellbeing, 1(2), 255-265. http://dx.doi.org/10.5502/ijw.v1i2.7

Joshanloo, M., \& Weijers, D. (2013). Aversion to happiness across cultures: A review of where and why people are averse to happiness. Journal of Happiness Studies, 15(3), 717-735. http://dx.doi.org/10.1007/s10902-013-9489-9

PriceWaterhouseCoopers LLP. (2008). Building the case for wellness. https://www.gov.uk/government/uploads/system/uploads/attachment data/file/209547/hwwb-dwpwellness-report-public.pdf

Robertson, I. T., Birch, A. J., \& Cooper, C. L. (2012). Job and work attitudes, engagement and employee performance: Where does psychological well-being fit in? Leadership \& Organization Development Journal, 33(3), 224-232. http://dx.doi.org/10.1108/01437731211216443

Schaufeli, W. B., Salanova, M., González-Romá, V., \& Bakker, A. B. (2002). The measurement of engagement and burnout: A two sample confirmatory factor analytic approach. Journal of Happiness Studies, 3(1), 71-92. http://dx.doi.org/10.1023/A:1015630930326

Schueller, S. M. (2012). Personality fit and positive interventions: Extraverted and introverted individuals benefit from different happiness increasing strategies. Psychology, 3(12A), 1166-1173. http://dx.doi.org/10.4236/psych.2012.312A172

Schulte, P., \& Vainio, H. (2010). Well-being at work: Overview and perspective. Scandinavian Journal of Work, Environment \& Health, 36(5), 422-429. http://dx.doi.org/10.5271/sjweh.3076

Seligman, M. E., \& Csikszentmihalyi, M. (2000). Positive psychology: An introduction. American Psychologist, 55(1), 5. http://dx.doi.org/10.1037/0003-066X.55.1.5

Sousa-Poza, A., \& Sousa-Poza, A. A. (2000). Well-being at work: A cross-national analysis of the levels and determinants of job satisfaction. Journal of Socio-Economics, 29(6), 517-538. http://dx.doi.org/10.1016/S1053-5357(00)00085-8

Spence, G. B. (2015). Workplace wellbeing programs: If you build it they may NOT come...because it's not what they really need! International Journal of Wellbeing, 5(2), 109-124. http://dx.doi.org/10.5502/ijw.v5i2.7

Vickerstaff, S., Phillipson, C., \& Wilkie, R. (2012). Work, health and wellbeing: The challenges of managing health at work. Bristol, United Kingdom: The Policy Press. 
Waddell, G., \& Burton, A. K. (2006). Is work good for your health and well-being? Retrieved from London: The Stationery Office.

Warr, P. (1990). The measurement of well-being and other aspects of mental health. Journal of Occupational Psychology, 63, 193-210. http://dx.doi.org/10.1111/j.2044-8325.1990.tb00521.x

Warr, P. (1992). Age and occupational well-being. Psychology and Aging, 7(1), 37-45.

http://dx.doi.org/10.1037/0882-7974.7.1.37

Wood, S. (2008). Job characteristics, employee voice and well-being in Britain. Industrial Relations Journal, 39(2), 153-168. http://dx.doi.org/10.1111/j.1468-2338.2007.00482.x

World Health Organisation (2005). The Bangkok Charter for Health Promotion in a Globalized World. Paper presented at the 6th Global Conference on Health Promotion, Bangkok, Thailand.

Young, V., \& Bhaumik, C. (2011). Health and well-being at work: A survey of employers. Sheffield, United Kingdom: Department for Work and Pensions. 


\section{Appendix A: Complete list of the elements offered in the BGL wellbeing programme}

\section{Current elements in the wellbeing programme}

At desk massage (limited availability)

Dental/hospital cash plan

Discounted gym membership

Employee Assistance Programme

Eyecare vouchers

Free fruit

Health screening

Healthy eating options in restaurant

Know your numbers (in-house health checks)

Organised lunchtime walking

Osteopath

Private medical insurance

Remedial sports massage

Second opinion medical referral

Weightwatchers site meetings

\section{Elements being considered for future inclusion in the programme}

Aerobic exercise classes at work

Ageing

Alcohol abuse education

Anger management

At desk massage (at all sites)

Building personal resilience

Changing facilities so it's easier to run/cycle to/from work

Coping with change

Driver fatigue

Drug abuse education

Energy

Fatigue

Health checks (blood pressure, weight, cholesterol, blood sugar)

Healthy back programme

Healthy eating programme

Healthy lifestyle

Managing anxiety

Organised team sports

Personal financial education

Quitting smoking help

Sleep clinic

Staying healthy on shift

Stress management support

Subsidised gym membership

Walking club

Weight management

Winter health (flu jabs, beating colds)

Work-life balance 